\title{
Characterization of Common Microscopy Artifacts with the Real Time Microscope
}

\author{
Michelle R. Gal*, Alexandra Mohr* Bruno Chue*, Nhu-An Pham*, Timothy M. Richardson* \\ *Richardson Technologies Inc., 20 Simpson Road, Bolton ON L7E 1G9 CANADA
}

\begin{abstract}
The Real Time Microscope (RTM) has the ability to provide high contrast images of living cellular systems as they have never been seen by previous light microscope systems. Preparation of samples for the RTM presents challenges in that common microscopy artifacts, such as dust and scratches on microscope slides and cover slips, are also seen in high contrast (Figures 1 and 2). Although they can be virtually undetectable when using standard light microscopes, the enhanced contrast of the RTM reveals all the details of such contaminants, which appear as dark spots, lines, unfocused hazes or diffraction ring patterns (Figures 3 and 4 ) in the image of interest.
\end{abstract}

In fluorescence microscopy, it is common to observe autofluorescent debris, non-specific binding of fluorophores to debris, contaminants and particles in fluorescent probe solutions and fluorophores that settle into defects on the slide or cover slip surfaces. Any of these unavoidable effects can skew results of image analysis by contributing to the overall background signal or by introducing discrete shapes into an image. The ability to view an RTM-fluorescence image in context with a corresponding RTM mode image is a powerful tool in reducing analysis error, not only by positively identifying artifacts, but also by preventing relevant information from being discarded (Figure 5).

This study provides a library of RTM and RTM-fluorescence images of common artifacts so they can be easily identified by RTM operators. In addition to images of dust and scratches on microscope slides and cover slips, as supplied by major manufacturers, solution precipitates, undissolved reagents, glove powder and other artifacts that are introduced during specimen preparation are also characterized using the RTM . Images of artifacts are shown at relevant magnification and gain settings, in their original states, and as they appear in typical wet specimen preparations. Proper methods for reagent, probe, media and sample preparation are discussed.

In providing this reference library, both RTM and conventional microscopists are assisted in obtaining the best possible images and understanding what their images illustrate. The goal is to reduce the possibility of image misinterpretation by familiarizing the microscopist with the appearance of standard and RTM-visible artifacts and debris. This paper is intended to assist in preventing microscopists from making embarrassing mistakes, such as declaring that glove powder artifacts are a newly discovered component of cytoplasm, or more importantly, keeping a microscopist from casually dismissing the "secret of life" as "just another artifact". 


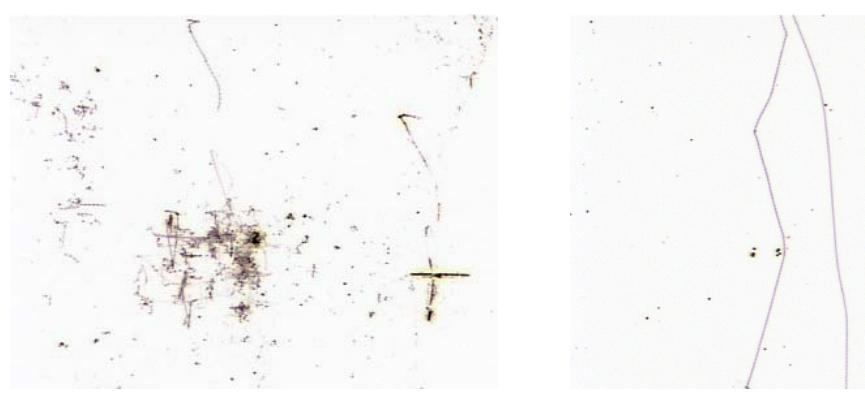

Figures 1 and 2: Scratches and debris found on a new microscope slide (field dimensions: $700 \mu \mathrm{m} \times 930 \mu \mathrm{m})$.
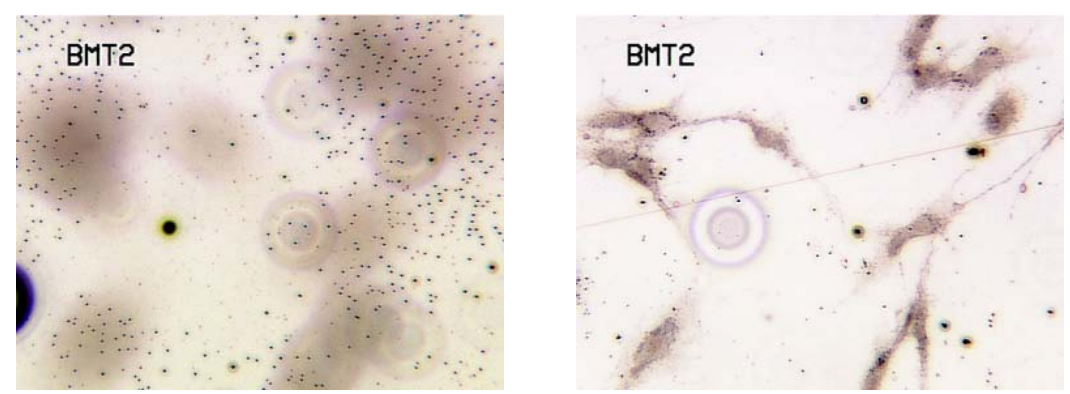

Figure 3: Preparation of fibroblasts on a glass chamber slide with cover slip showing (a) debris on cover slip at cover slip focal plane which results in (b) diffraction ring pattern at focal plane of cells, also shows a scratch across the field of view (field dimensions: $350 \mu \mathrm{m}$ x $460 \mu \mathrm{m}$ ).
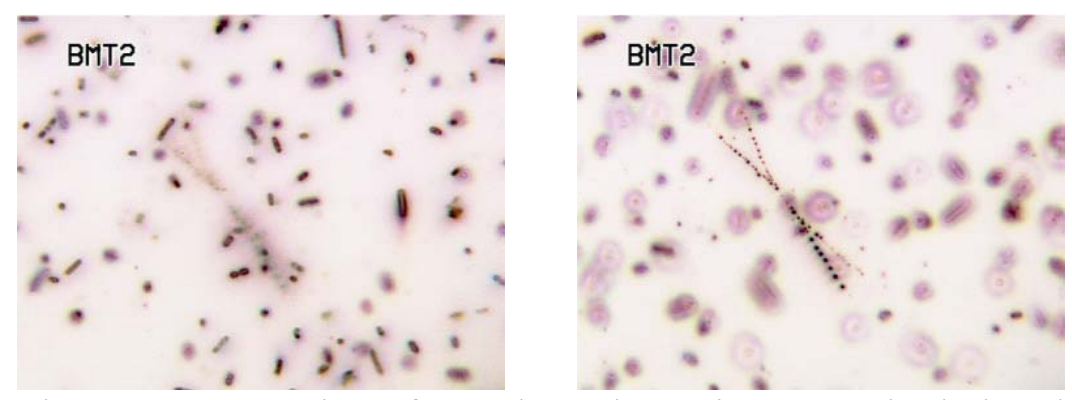

Figure 4: Preparation of sauerkraut bacteria on standard ultraclean glass slide with cover slip, showing (a) dark haze in bacteria focal plane originating from (b) an X-shaped defect on slide surface (field dimensions: $53 \mu \mathrm{m} \times 70 \mu \mathrm{m}$ ).
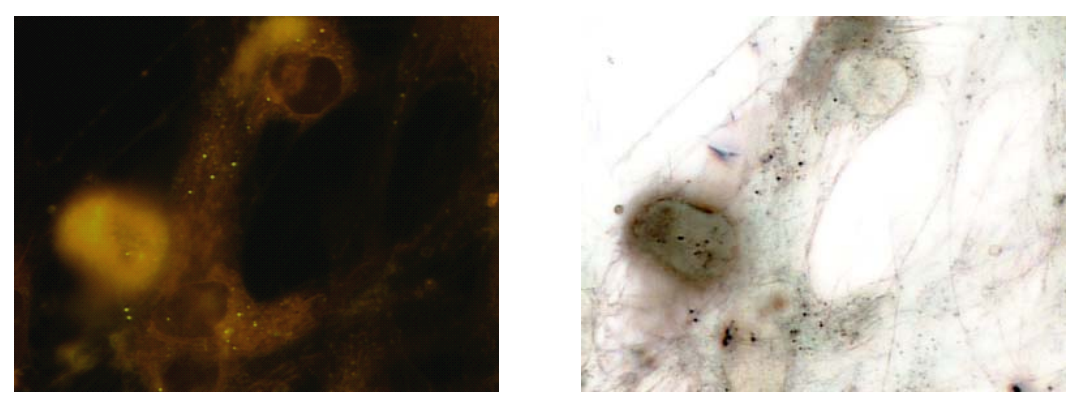

Figure 5: Preparation of fibroblasts stained with nile red fluorescent probe. (a) The RTMfluorescent mode image, showing fluorescent signal in distinctly cell-like patterns as well as an unfocused haze in the lower left corner. (b) The corresponding RTM mode image illustrates that the haze originates from a rounded, unhealthy cell (field dimensions: $90 \mu \mathrm{m}$ x $120 \mu \mathrm{m}$ ). 